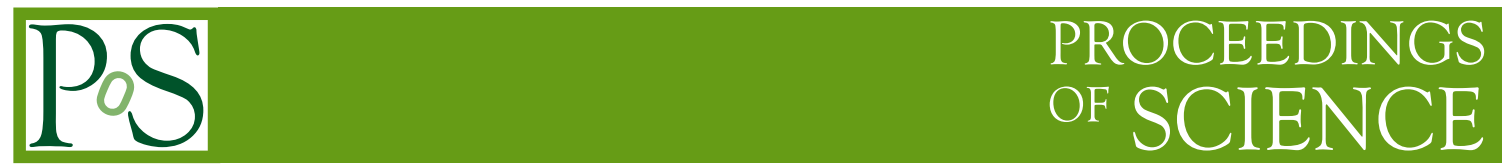

\title{
Analysis of $\mathbf{b}$ jets in $\mathbf{p}-\mathrm{Pb}$ collisions at $\sqrt{s_{\mathrm{NN}}}=5 \mathrm{TeV}$ with ALICE
}

\section{Isakov Artem* for the ALICE Collaboration}

Nuclear Physics Institute of the Czech Academy of Sciences, Rez, Czechia

E-mail: isakoveujf.cas.cz

We report on the measurement of inclusive $p_{\mathrm{T}}$ spectrum of charged $\mathrm{b}$ jets in $\mathrm{p}-\mathrm{Pb}$ collisions at $\sqrt{s_{\mathrm{NN}}}=5.02 \mathrm{TeV}$ by ALICE. We describe details of the analysis with an emphasis on the performance of the secondary vertex tagging. The fully corrected $b$-jet spectrum is compatible with calculation of the POWHEG HVQ tune.

European Physical Society Conference on High Energy Physics - EPS-HEP2019 -

10-17 July, 2019

Ghent, Belgium

*Speaker. 


\section{Introduction}

The Quark-Gluon Plasma (QGP) [1] is a state of strongly-interacting matter where color charges get deconfined. Large energy densities and temperatures which are necessary to produce the hot QGP are achieved by colliding ultra-relativistic heavy nuclei [2]. The produced QGP rapidly expands, cools and transforms to the hadron gas. Hence, to study properties of the QGP, we need to choose convenient probes. Since the $\mathrm{b}$ quark has a large mass, its production takes place mostly in initial hard scatterings and it is calculable using perturbative Quantum Chromodynamics (QCD). Thus, beauty quarks can be considered as ideal self-generated penetrating probes of the created medium and utilized to investigate mass-dependent parton energy loss in the hot QCD medium or in cold nuclear matter (CNM) [2]. The measurement of beauty-jet production in $\mathrm{p}-\mathrm{Pb}$ collisions is fundamental for the investigation of CNM effects, such as the modification of Parton Distribution Functions in nuclei with respect to protons or energy loss in the CNM. Understanding these effects is necessary for the proper interpretation of results obtained in $\mathrm{Pb}-\mathrm{Pb}$ collisions.

\section{ALICE experiment}

A Large Ion Collider Experiment (ALICE) [3] is one of the four major experiments at the Large Hadron Collider. It is focused on the study of the QGP created in heavy-ion collisions. ALICE provides a broad variety of detector systems for measurements of hadrons, leptons, photons in a wide kinematic range down to very low $p_{\mathrm{T}}$. The present analysis uses data recorded in the Inner Tracking System (ITS) and the Time Projection Chamber (TPC), both of which are in the central barrel region, immersed in a magnetic field of 0.5 Tesla. These detectors cover the full azimuth and pseudorapidity $|\eta|<0.9$. The excellent tracking capabilities of ALICE provides a track impact parameter resolution better than $70 \mu \mathrm{m}$ at $p_{\mathrm{T}}>1 \mathrm{GeV} / c$ [4]. This enables to efficiently reconstruct secondary decay vertices of $\mathrm{D}$ or $\mathrm{B}$ hadrons which are displaced hundreds of micrometers from the primary interaction vertex $(\mathrm{PV})$.

\section{3. b-jet candidate reconstruction}

We have analyzed minimum bias $\mathrm{p}-\mathrm{Pb}$ collisions at $\sqrt{s_{\mathrm{NN}}}=$ 5.02 TeV recorded by ALICE in 2016. Details of event and track selection can be found in [5]. Jet reconstruction was based on the FASJET anti- $k_{\mathrm{T}}$ algorithm [6] with a resolution parameter of $R=0.4$ using the $p_{\mathrm{T}}$-recombination scheme. The tracks of charged particles with $p_{\mathrm{T}}>0.15 \mathrm{GeV} / c$ were included in the jets. The pseudorapidity of the jets was constrained to $\left|\eta_{\text {jet }}\right|<$ 0.5 . The $p_{\mathrm{T}}$ spectrum of the jets was corrected for the underlying event density with the method described in [7].

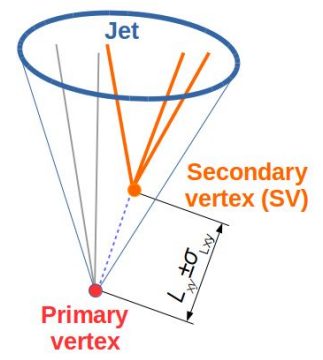

Figure 1: Scatch of the SV reconstruction

Identification of b-jet candidates was based on reconstruction of displaced secondary vertices (SV) from b-hadron decays. These secondary vertices were constructed from triplets of jet constituents (see Fig. 1). In further analysis, topological constraints on the most displaced SV found in the jet were imposed to suppress admixture of light-flavor and c-quark jets. 
The first requirement puts an upper limit $\sigma_{\mathrm{SV}}^{\max }$ on the size of SV resolution. The resolution was calculated as $\sigma_{\mathrm{SV}}=\sqrt{\sum_{i=1}^{3} d_{i}^{2}}$, where $d_{i}$ denotes the distance of closest approach of each jet constituent to the SV. In the analysis, $\sigma_{\mathrm{SV}}^{\max }$ was varied from 0.02 to $0.05 \mathrm{~cm}$ with a step of $0.01 \mathrm{~cm}$.

The second selection criterion, which was applied to the SV, constrained the significance of SV displacement from the PV: $S L_{x y}=L_{x y} / \sigma_{L_{x y}}$. Here $L_{x y}$ is the transverse distance between the $\mathrm{PV}$ and $\mathrm{SV}$ and $\sigma_{L_{x y}}$ is the corresponding uncertainty. For b-jet candidates, the significance was required to be larger than a chosen cutoff $S L_{x y}^{\min }$. In the present analysis, $S L_{x y}^{\min }$ was varied from 5 to 9 with a step of 1 .

\section{Purity and efficiency of the SV tagging method}

The selected sample of $b$ jets was corrected for the efficiency of SV tagging and for the remaining admixture of $\mathrm{c}$ jet and light-flavor jets as follows:

$$
N_{\mathrm{bjet}}^{\mathrm{corr}}\left(p_{\mathrm{T}, \text { jet }}^{\mathrm{ch}, \text { reco }}\right)=N_{\mathrm{b} \text { jet }}^{\mathrm{raw}}\left(p_{\mathrm{T}, \text { jet }}^{\mathrm{ch} \text { reco }}\right) \times \frac{P_{\mathrm{b}}\left(p_{\mathrm{T}, \text { jet }}^{\text {ch, reco }}\right)}{\varepsilon_{\mathrm{b}}\left(p_{\mathrm{T}, \text { jet }}^{\text {ch, reco }}\right)},
$$

where $P_{\mathrm{b}}$ and $\varepsilon_{\mathrm{b}}$ are b-jet tagging purity and efficiency for the chosen $S L_{x y}$ and $\sigma_{\mathrm{SV}}$ selection. Both the purity and the efficiency depend on the transverse momentum of the jet, corrected for the estimated underlying event density density $\left(p_{\mathrm{T}, \text { jet }}^{\text {ch, reco }}\right)$.

The efficiency of SV tagging for jets with different flavours was estimated using simulated $\mathrm{p}-\mathrm{Pb}$ collisions at $\sqrt{s_{\mathrm{NN}}}=5.02 \mathrm{TeV}$ obtained by embedding PYTHIA 6 event [8] into EPOS [9] events. The efficiency gives the probability that a jet with a given flavor will pass the imposed SV tagging

criteria:

$$
\varepsilon_{\text {flavor }}\left(p_{\mathrm{T}, \text { jet }}^{\text {ch, reco }}\right)=\frac{N_{\text {flavor }}^{\text {selected }}}{N_{\text {flavor }}^{\text {all }}},
$$

where $N_{\text {flavor }}^{\text {all }}$ defines the number of jets with specific flavor that were reconstructed without any constraint on the parameters of SV and $N_{\text {flavor }}^{\text {select }}$ is the number of jets with the same flavor that were obtained for a given SV selection. In Fig. 2, we present the SV tagging efficiencies for the default choice of SV selection criteria $\left(S L_{x y}>7, \sigma_{\mathrm{SV}}<0.03 \mathrm{~cm}\right)$.

The purity of the selected b-jet sample is de-

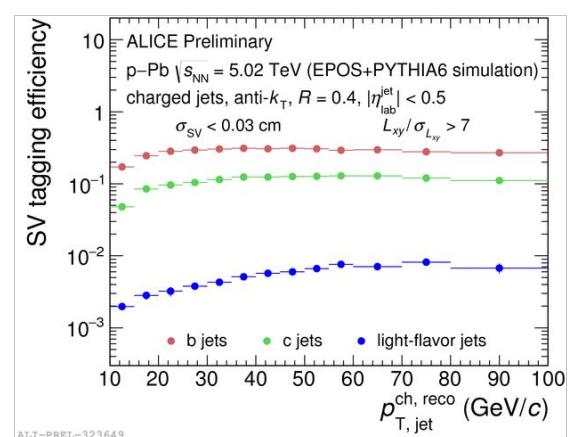

Figure 2: SV tagging efficiency of jets with different flavors fined as the fraction of $b$ jets among all selected jets. It was estimated with a data driven template fit method and method called the POWbc, both of which are now discussed briefly.

The data-driven template fit method is a statistical method which estimates purity of the selected b-jet candidate sample based on the invariant mass distribution of the reconstructed SV. The invariant mass distributions corresponding to a given $p_{\mathrm{T} \text {, jet }}^{\text {ch } \text {,eco }}$ bin is parameterized by a linear combination of three invariant mass templates $T_{\mathrm{b}}, T_{\mathrm{c}}, T_{\mathrm{LF}}$ corresponding to $\mathrm{b}$ jets, $\mathrm{c}$ jets and light flavor jets

$$
n_{\mathrm{SV}}=P_{\mathrm{b}} \cdot T_{\mathrm{b}}+P_{\mathrm{c}} \cdot T_{\mathrm{c}}+P_{\mathrm{LF}} \cdot T_{\mathrm{LF}}
$$


Provided that the integral of the templates are normalized to unity, the coefficient $P_{\mathrm{b}}$ gives the purity of the b-jet sample. The templates were obtained from the combined PYTHIA 6 and EPOS events. The optimal values of the purities were obtained from a fit using the TMinuit package [10] by minimising the $\chi^{2}$ criterion:

$$
\chi^{2}=\sum \frac{\left(n_{\mathrm{SV}}-P_{\mathrm{b}} \cdot T_{\mathrm{b}}-P_{\mathrm{c}} \cdot T_{\mathrm{c}}-P_{\mathrm{LF}} \cdot T_{\mathrm{LF}}\right)^{2}}{\sigma_{n_{\mathrm{SV}, \mathrm{i}}}^{2}+\left(\sigma_{T_{\mathrm{b}, \mathrm{i}}} \cdot P_{\mathrm{b}}\right)^{2}+\left(\sigma_{T_{\mathrm{c}, \mathrm{i}}} \cdot P_{\mathrm{c}}\right)^{2}+\left(\sigma_{T_{\mathrm{c}, \mathrm{i}}} \cdot P_{\mathrm{c}}\right)^{2}},
$$

where $\sigma_{n_{\mathrm{SV}, \mathrm{i}}}$ is the statistical uncertainty on the measured $n_{\mathrm{SV}}$, the spectrum of the secondary vertex invariant mass. The statistical uncertainties $\sigma_{T_{\mathrm{b}, \mathrm{i}}}, \sigma_{T_{\mathrm{c}, \mathrm{i}}}, \sigma_{T_{\mathrm{LF}, \mathrm{i}}}$ correspond to each jet flavor and are obtained from the MC templates. An illustration of the fit is shown on the left panel of Fig. 3. The data driven template fit method fails to provide reliable fits for $p_{\mathrm{T} \text {, jet }}^{\mathrm{ch}, \text { reco }}>35 \mathrm{GeV} / c$, where the measured invariant mass distributions suffer from poor statistics.
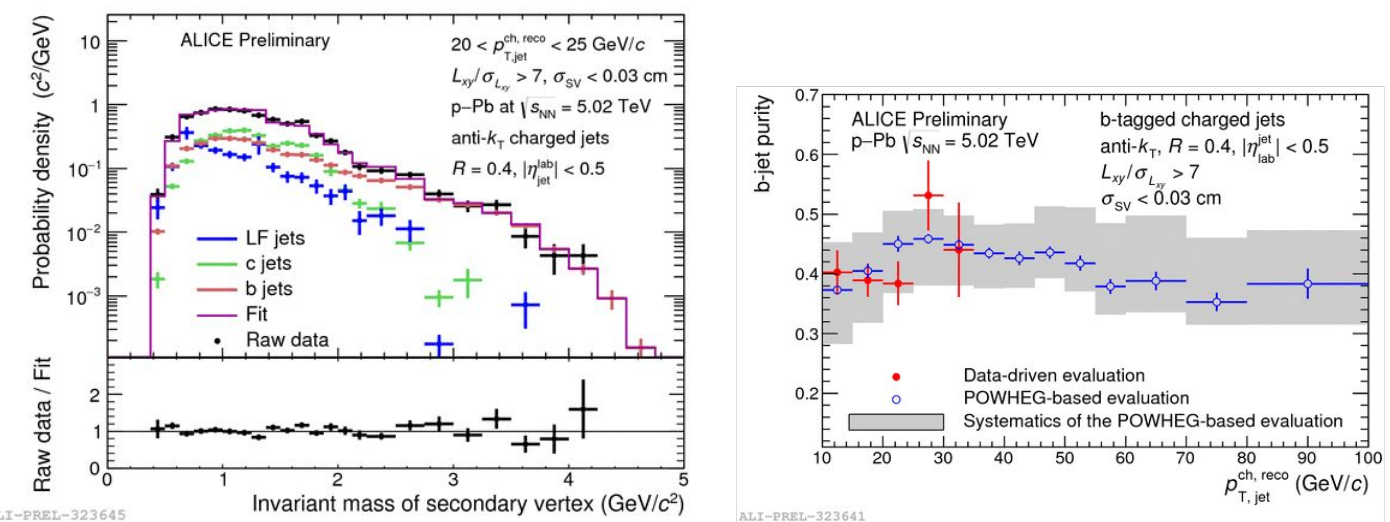

Figure 3: Left: Invariant mass distribution of the default cuts and the corresponding fit with MC templates. Right: b-jet purities obtained with the data-driven method and the POWbc method for the optimal choice of POWHEG settings.

The POWbc method overcomes the problem of the data-driven method. In this approach, particle-level $p_{\mathrm{T}}$ spectra of $\mathrm{b}$ and $\mathrm{c}$ jets generated with the POWHEG [11] generator were folded with a response matrix that accounts for jet $p_{\mathrm{T}}$ smearing due to local background fluctuations and detector effects. Subsequently, the raw spectrum of light-flavor jets was obtained by subtracting the POWHEG raw spectra of $b$ and $c$ jets from the measured raw inclusive jet spectrum. The purity of the selected b-jet sample was then estimated using the formula:

$$
P_{\mathrm{b}}=\frac{N_{\mathrm{b}} \cdot \varepsilon_{\mathrm{b}}}{N_{\mathrm{b}} \cdot \varepsilon_{\mathrm{b}}+N_{\mathrm{c}} \cdot \varepsilon_{\mathrm{c}}+N_{\mathrm{LF}} \cdot \varepsilon_{\mathrm{LF}}},
$$

where $N_{\mathrm{b}}, N_{\mathrm{c}}, N_{\mathrm{LF}}$ denote the raw inclusive $p_{\mathrm{T}}$ spectra of different jet flavors and $\varepsilon_{\mathrm{b}}, \varepsilon_{\mathrm{c}}, \varepsilon_{\mathrm{LF}}$ are the associated $\mathrm{SV}$ tagging efficiencies.

This method, however, depends on the particular setting of the POWHEG generator (regularization scale, renormalization scale, quark masses). The purities obtained by the data-driven technique were therefore used to constrain the plausible set of POWHEG settings by imposing a cut on the following quantity: 


$$
\chi^{2}=\sum \frac{\left(P_{\mathrm{b}}^{\text {POWbc }}-P_{\mathrm{b}}^{\text {data-driven }}\right)^{2}}{\sigma_{\text {POWbc }}^{2}+\sigma_{\text {data-driven }}^{2}} .
$$

Here the sum runs over all $p_{\mathrm{T}}$ bins across all $S L_{x y}^{\min }$ and $\sigma_{\mathrm{SV}}$ settings. $P_{\mathrm{b}}^{\mathrm{x}}$ and $\sigma_{\mathrm{x}}$ is the b-jet purity and the corresponding uncertainty obtained by the method for $\mathrm{x}=$ POWbc or data-driven. Acceptable POWHEG settings have $\chi^{2} /$ n.d.f. $<10$. Purities obtained by the data driven template fit method and the POWbc method are shown on the right panel of Fig. 3. The gray band represents the resulting uncertainty attributed to the plausible choices of POWHEG settings.

\section{Results}

After correcting the raw $p_{\mathrm{T}}$ spectrum of $\mathrm{b}$-jet candidates for SV tagging efficiency and $\mathrm{b}$ jet purity with Eqn. 4.1, the b-jet spectrum was corrected for momentum smearing due to local background fluctuations $\left(\delta p_{\mathrm{T}}\right)$ and detector effects by means of the SVD unfolding [13]. The response matrix used in the unfolding procedure was generated with the PYTHIA $6+$ EPOS MC simulation with the transport code GEANT3 [12]. The fully corrected $p_{\mathrm{T}}$ spectrum of $\mathrm{b}$ jets is shown in Fig. 4. The cross section is measured down to $10 \mathrm{GeV} / \mathrm{c}$. Within the quoted statistical and systematic uncertainties, the data agrees with the POWHEG HVQ [14] predictions.

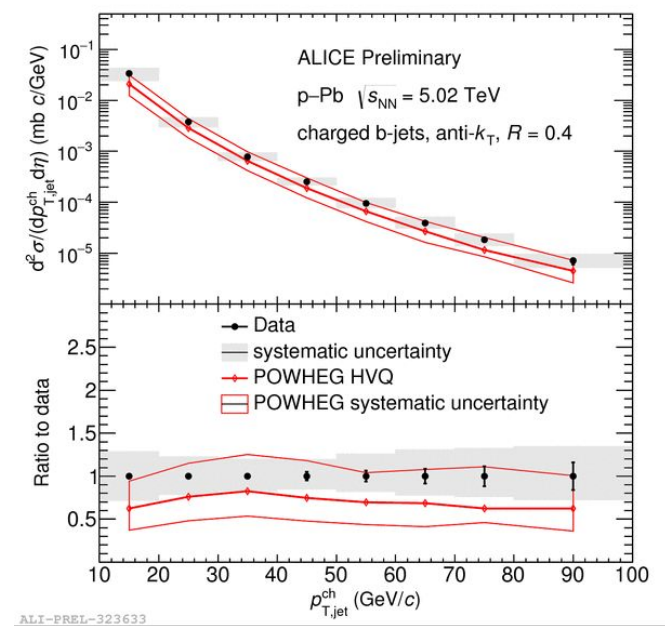

Figure 4: Fully corrected inclusive $p_{\mathrm{T}}$ spectrum of charged anti- $k_{\mathrm{T}} \mathrm{b}$ jets with $R=0.4$ obtained by the SV tagging method. The data are compared with the POWHEG HVQ tune with the PYTHIA 6 fragmentation and the EPS09NLO parton distribution functions. Systematic uncertainty bars on the data are marked by gray boxes. Systematic uncertainty bars of POWHEG simulations come from the variation of the simulation parameters

In summary, we have used the SV tagging method to measure $p_{\mathrm{T}}$ spectrum of charged $\mathrm{b}$ jets with anti- $k_{\mathrm{T}}$ algorithm. We propose that measuring the $\mathrm{b}$-jet spectrum using the same method in $\mathrm{pp}$ collisions will enable determination of the nuclear modification factors $R_{\mathrm{pPb}}$ for b jets.

\section{References}

[1] E. Shuryak, Physics of Strongly coupled Quark-Gluon Plasma, Prog.Part.Nucl.Phys. 62, 48-101 (2009), arXiv:0807.3033 [hep-ph]. 
[2] R. Stock, Relativistic Nucleus-Nucleus Collisions and the QCD Matter Phase Diagram, arXiv:0807.1610v1 nucl-ex.

[3] K. Aamodt et al. (ALICE Collaboration), The ALICE experiment at the CERN LHC, JINST 3, S08002 (2008).

[4] B. Abelev et al. (ALICE collaboration), Performance of the ALICE Experiment at the CERN LHC, Int. J. Mod. Phys A29, 1430044 (2014).

[5] S. Acharya et al. (ALICE collaboration), Constraints on jet quenching in $p-P b$ collisions at $\sqrt{s_{\mathrm{NN}}}=5.02 \mathrm{TeV}$, Phys. Lett. B783, 95-113 (2018).

[6] M. Cacciari et al., FastJet user manual, Eur. Phys. J. C72, 1896 (2012) .

[7] S. Chatrchyan et al. (CMS Collaboration), Measurement of the underlying event activity in pp collisions at $\sqrt{s}=0.9$ and $7 \mathrm{TeV}$ with the novel jet-area/median approach, JHEP 08, 130 (2012)

[8] T. Sjostrand et al., PYTHIA 6.4 Physics and Manual, JHEP 0605, 026 (2006) hep-ph/0603175.

[9] B. Guiot, K. Werner, Hard probes and the event generator EPOS, J.Phys.Conf.Ser. no.1., 589 (2015)

[10] F. James, MINUIT Function Minimization and Error Analysis, CERN-D-506.

[11] C. Oleari, The POWHEG-BOX, Nucl.Phys.Proc.Suppl. 205-206, 36-41 (2010).

[12] R. Brun et al. GEANT3, CERN-DD-EE-84-1

[13] A. Hoecker, V. Kartvelishvili, SVD Approach to Data Unfolding, Nucl.Instr.Meth.Phys.Res. A372, 469-481 (1996).

[14] S. Frixione et al., The POWHEG-hvq manual version 1.0 BICOCCA-FT-07-10, arXiv:0707.3081 